\title{
Electron Beam-Induced Sample Contamination in the SEM
}

\author{
A. E. Vladár and M. T. Postek
}

National Institute of Standards and Technology*, Gaithersburg, MD 20899 M/s. 8212

Electron beam induced contamination, i.e. the deposition of carbonaceous material over the sample surface bombarded by the electron beam is almost always present after viewing in the scanning electron microscope (SEM). This is one of the most troublesome problems in high-resolution scanning electron microscopy since it induces physical change the actual structure of the material being viewed, generally by obscuring the fine detail. Its importance is growing, especially for nanometer resolution imaging, where small surface structures can easily be buried in even few seconds. Therefore, it is essential to find the origins of electron beam induced contamination and regularly measure it and keep it at acceptable levels.

Several published studies $[1,2,3]$ have found that the origins of the contamination are both the sample itself and the vacuum in the SEM. Although the pumping system is a major contributor to this problem, the history of the specimen prior to entering the vacuum system must also be considered relative to the deposition of hydrocarbon contamination. All this leads to problems especially at low accelerating voltages where the carbonaceous layer deposited by the electron beam becomes extremely obvious. It is common that instruments with "good" vacuum will still exhibit unacceptable rates of electron beam induced contamination because of residual organic materials in the vacuum. (Figure 1.) Deposition rates of a few tens of nanometers per second have been observed, but smaller rates are more common especially with the more "modern" instruments. In many cases the presence of contamination is not obvious; it only leads to a change in the amount of electrons leaving the sample, hence a drop of signal. In other cases, surface contamination will lead to serious measurement errors and thus, will rule out collection of any useful data.

Reimer [4] describes a process of drift of large molecular weight molecules under the electron bombardment. The deposition of the material forming the contamination layer is a dynamic process. Molecules arrive at and leave the sample surface at the same time. The amount of contamination deposited depends on the electron dose, (i.e. the length of time the beam dwells on the sample and the beam current). The longer the dwell time, the thicker the contamination becomes.

In this study we report the results of contamination rate measurements, the dependence of the deposition of contaminants on electron beam current and landing energy and sample composition. We also report the results effective cleaning methods that were successfully used to diminish electron beam induced sample contamination. For sample cleaning a Model 1020 Plasma Cleaner** from Fischione Instruments [5] was used and for cleaning of the SEM sample chamber an Evactron ${ }^{\circledR}$ RF Plasma Cleaner - Anti-Contaminator unit from XEI Scientific [6] was used. The SEMs used in this study were high vacuum and environmental type microscopes. These results were obtained both before and after cleaning of the SEM and samples. 


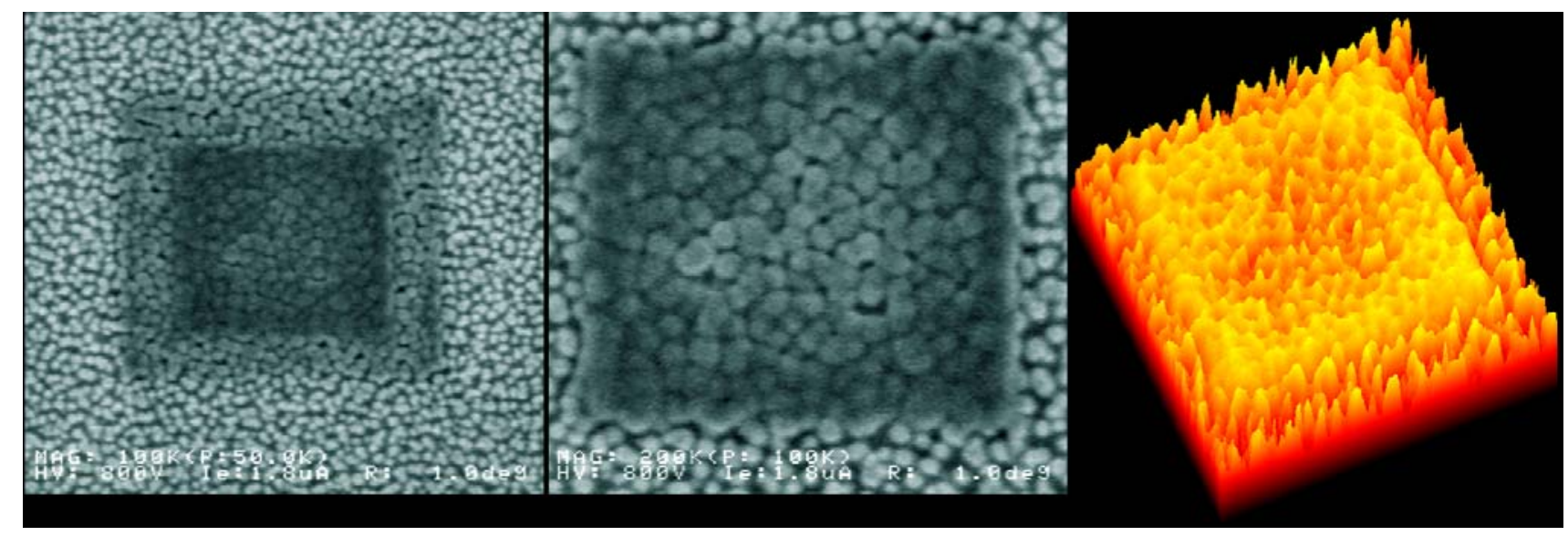

Figure 1. Contamination layers formed during 3 minutes (at $75 \mathrm{kx}$ magnification) and 6 minutes (at $100 \mathrm{kx}$ magnification) of continuous imaging. CD-SEM, $800 \mathrm{~V}$ accelerating voltage, 3 pA beam current, $2 \times 10^{-4} \mathrm{~Pa}$ vacuum. The image on the left was taken at $50 \mathrm{kx}$ magnification at the end of the bombardment, the middle and the left (isometric view) images show the center thick contamination region.

The findings of this study point to the need of regular contamination rate measurements and recommend methods for these and offer effective ways for fighting electron beam induced contamination.

\section{References}

[1] A. Vladár, M Postek, and R. Vane. Active Monitoring and Control of Electron-beam-induced Contamination. Proc. SPIE 2001 4344:835-843.

[2] M. T. Postek. An Approach to the Reduction of Hydrocarbon Contamination in the SEM. SCANNING 1996.18:269-274

[3] N. Sullivan, T. Mai, S. Bowdoin and R. Vane. A Study of the Effectiveness of the Removal of Hydrocarbon Contamination by Oxidative Cleaning Inside the SEM. Microscopy \& Microanalysis 2002Vol. 8, Supplement 2, 720CD.

[4] L. Reimer. Image Formation in Low-Voltage Electron Microscopy SPIE Opt. Eng. Press; Volume TT 12 (1993) ISBN 0-8194-1206-6

[5] http://www.fischione.com/products/model_1020.asp

[6] http://www.evactron.com/index.html

*Contribution of the National Institute of Standards and Technology, not subject to copyright.

** Certain commercial equipment is identified in this report to adequately describe the experimental procedure. Such identification does not imply recommendation or endorsement by the National Institute of Standards and Technology, nor does it imply that the equipment identified is necessarily the best available for the purpose. 